\title{
Single versus combination therapy in acute tocolysis: A prospective randomized controlled trial*
}

\author{
Wafa R. Al-Omari ${ }^{1 \#}$, Muzibunnisa A. Begam ${ }^{1}$, Farsana S. Khan ${ }^{1}$, Iman Y. Khudhair ${ }^{1}$, \\ Nicolaas J. Nagelkerke ${ }^{2}$ \\ ${ }^{1}$ Department of Obstetrics \& Gynecology, Tawam/Johns Hopkins Hospital, Al Ain, UAE \\ ${ }^{2}$ Department of Community Medicine, Faculty of Medicine and Health Sciences, UAE University, Al Ain, UAE \\ Email: " wafa_alomari@yahoo.com, drmuzis@yahoo.com, drfarsana@hotmail.com, imankhudhair@yahoo.com, \\ nico.nagelkerke@uaeu.ac.ae
}

Received 24 January 2013; revised 26 February 2013; accepted 7 March 2013

\begin{abstract}
This is a prospective controlled randomized trial conducted in 92 women with singleton pregnancies in preterm labor. The tocolytic efficacy and safety of combination atosiban and nifedipine was compared with that of the single agent, atosiban. Both lines of intervention was administered for 48 hours. Progression of labor was assessed by the frequency of uterine contractions and cervical changes. For statistical purpose, intent-to-treat (ITT) analysis was used throughout. Efficacy, as determined by the proportion of women in each group who did not deliver after therapy initiation, was comparable with no significant differences, at $48 \mathrm{hrs}(\mathbf{9 1 . 5 \%}$ vs $\mathbf{9 1 . 1 \% )}$ and at 7 days $(90.7 \%$ vs $85.7 \%)$ for the atosiban and the combination groups respectively. Safety was assessed by the numbers of adverse events. Maternal side effects were reported more in the combination group ( $34 \%$ vs $64 \% ; P=0.006)$. Perinatal outcomes were similar between the groups. We conclude that the addition of nifedipine did not substantially improve the clinical outcomes beyond that were achieved with atosiban alone. Moreover, it has increased maternal side effects. Future research could focus on combination of other tocolytics.
\end{abstract}

Keywords: Atosiban; Combination Tocolysis; Preterm Labor

\section{INTRODUCTION}

Preterm birth, defined as birth at less than $37+0$ weeks of gestation, accounts for $5 \%$ to $11 \%$ of births in the world [1]. But, it represents the single largest cause of

\footnotetext{
*Name of the trial registry: www.clinicaltrials.gov, Registry No: NCT01429545 .

Disclosure of Interests: None.

Funding: None.

\#Corresponding author.
}

mortality and morbidity for newborns and a major cause of morbidity for pregnant women [2]. A multi-level modeling of hospital service utilization of preterm birth has outlined the huge economic consequences in the first 10 years of life [3]. Neonatal mortality has declined, mostly due to improved management of the very low birth weight babies rather than prevention of preterm labor (PTL).

The most common treatment for PTL involves pharmacological inhibition of preterm uterine contractions. The choice of tocolytic agent depends on its ability to delay the delivery by at least 48 hours and preferably longer from the time of administration of steroids without maternal or fetal side effects [4]. There is considerable variation in the type of tocolytic agent used. Single agent tocolysis using ritodrine ( $\beta$-agonist), atosiban (oxytocin antagonist) or nifedipine (calcium channel blocker) is a common practice. Meta analysis from Cochrane systematic review failed to demonstrate the superiority of atosiban over betamimetics or placebo in terms of tocolytic efficacy or infant outcomes, but, the maternal drug reactions were fewer with atosiban [5]. Nifedipine is the only agent associated with improved perinatal outcomes and fewer maternal side-effects than betamimetics [6]. Atosiban and nifedipine have been shown to have equal efficacy, however maternal side-effects were more pronounced with nifedipine $[7,8]$.

Due to the differences in their pharmacokinetics and pharmacodynamics, one might expect to have improved tocolysis when two agents are combined. In vitro studies have demonstrated that simultaneous blockade of these different pathways could result in an additive or even synergistic effect capable of producing better uterine relaxation than induced by each drug alone $[9,10]$. Accordingly, the use of multiple agent therapy has been suggested as a way forward in tocolytic research $[9,11]$. In an observational study, combination therapy without serious side effects has been used in the management of PTL at early gestational ages [12]. However, this was 
not tested in any structured human trials.

The objective of this study was to compare the tocolytic efficacy and safety of the combination of atosiban and nifedipine against the single agent, atosiban in PTL.

\section{MATERIALS \& METHODS}

This was a prospective randomized controlled clinical trial conducted at Tawam Hospital, Al Ain, United Arab Emirates (UAE), over a period of 3 years from April 2007-September 2010. The study protocols were approved by Al Ain Medical District Human Research Ethics Committee (Protocol Number of 06/95) and were conducted in accordance with good clinical practice and the Declaration of Helsinki. An informed consent was obtained from each patient prior to being enrolled into the study.

The inclusion criteria were; 1) singleton pregnancies with intact membranes, 2) gestational age of $24-34$ completed weeks, 3) clinical diagnosis of PTL (regular uterine contractions $>4$ per 30 minutes, each lasting for 30 seconds and cervical dilatation of $0-3 \mathrm{~cm}$ (nullipara) or 1 - $3 \mathrm{~cm}$ (multipara) with cervical effacement of $\geq$ $50 \%$; [13] and 4) $\geq 18$ yrs of age. The exclusion criteria were the presence of indication for delivery, ruptured membranes, multiple pregnancy, fetal distress, suspected chorioamnionitis, prior tocolytic therapy and patients refusal. Patients with gestational diabetes controlled on diet were included. Insulin-dependent diabetics and women with other chronic medical problems such as hypertension were all excluded.

Medical evaluation including thorough history, physical examination and pertinent investigations were performed. All women being less than 34 weeks of gestation received steroids as per our unit policy. Antibiotics were given only when clinically indicated. During the patient's evaluation, intravenous hydration with normal saline at a rate of $120 \mathrm{ml} / \mathrm{hr}$. after an initial bolus dose of $200 \mathrm{ml}$ was administered.

\subsection{Randomization}

A box containing 100 folded slips divided equally for single and combination therapy was maintained in our labor ward. Slips were picked blindly by the recruiting physician, patients were categorized into: group A (allocated to atosiban alone) and group B (simultaneous administration of atosiban and nifedipine). Atosiban was given as a bolus (6.7 $\mathrm{mg} \mathrm{IV}$ ) over $1 \mathrm{~min}$ then an infusion of $18 \mathrm{mg} / \mathrm{hr}$ for $3 \mathrm{hrs}$ followed by $6 \mathrm{mg} / \mathrm{hr}$ for 48 hours [14]. Nifedipine was given in the dose of $10 \mathrm{mg}$ orally every $15 \mathrm{~min}$ till uterine quiescence ( $<4$ contractions $/ \mathrm{hr}$ ) was achieved. Maximum dose was $40 \mathrm{mg}$ in the first hour followed by maintenance dose of $10 \mathrm{mg}$ every $6 \mathrm{hrs}$ for 48 hrs $[15,16]$.

The frequency of uterine contractions was monitored using external tocography until uterine quiescence $(<4$ contractions/hr) and subsequently every 4 hours till the completion of the therapy. Maternal and fetal monitoring was undertaken by regular assessment of maternal symptoms, vital signs and continuous cardiotocography.

Rescue tocolysis with salbutamol could be given if treatment with the study drug failed, due to either progression of labor or drug discontinuation because of side effects, as judged by the treating physician. Progression of PTL was assumed when any two of the following three criteria were met: a contraction rate of $\geq 4 / \mathrm{h}$, an increase in cervical dilatation of $\geq 1 \mathrm{~cm}$ or an increase in cervical effacement $\geq 25 \%$ from the initial assessment [17].

The primary outcomes in this study were efficacy and safety of the tocolysis. Tocolytic efficacy was assessed in terms of the proportion of women undelivered at 48 hours and seven days of initiation of therapy without the need for rescue tocolysis. Tocolytic effectiveness was assessed as total number of women undelivered during the same period even if rescue tocolysis was used.

Safety was assessed by maternal, fetal and neonatal adverse events. Particular emphasis was placed on serious maternal adverse outcomes such as cardiac arrest, respiratory arrest, admission to intensive care unit and death [11]. Maternal and fetal tachycardia was defined as a heart rate exceeding 120 and 170 beats per minute respectively. Perinatal complications were recorded by neonatal morbidity and mortality until discharge from the hospital.

Secondary outcome measures were: mean gestational age at delivery, mean infant birth weight, admission and length of stay at neonatal intensive care unit (NICU).

The primary and secondary outcomes were chosen based on The Canadian PTL Investigators Group [18].

\subsection{Statistical Analysis}

All statistical analyses were performed using SPSS version 19.

Standard statistical methods for comparing continuous and dichotomous outcomes between groups such as Fisher's exact test (2-sided) for $2 \times 2$ tables, trend test for cross-tabulations with ordinal categories, the MantelHaenszel (M-H) pooled Odds-Ratio and significance test for stratified analyses, the Mann-Whitney U test for continuous measurements, and the log-rank test to compare Kaplan-Meier survival curves were used. Intent-totreat (ITT) analysis was used throughout. Power calculation was not performed in this study and we depended on $95 \%$ confidence interval. 


\section{RESULTS}

A total of 92 women were recruited and randomized to receive either the control, atosiban (group $\mathrm{A}=47$ ) or the combination tocolysis, atosiban and nifedipine (group B $=45$ ). The study profile is shown in Figure 1 .

The baseline characteristics of the two groups were comparable, with no significant differences (Table 1).

\subsection{Primary Outcomes}

Rescue tocolysis was not used as labor progressed quickly in those patients for whom the study drug failed. Hence, the tocolytic effectiveness was the same as tocolytic efficacy.

In terms of tocolytic efficacy, there were no statistically significant differences between the two groups (Table 2). Also, further analysis after sub grouping into different gestational ages showed no statistically significant differences (Table 3). However, the combination group achieved uterine quiescence faster than single agent group, and the difference was significant (Table 2).

No serious side effects were encountered in either group. However, minor cardiovascular adverse events and development of multiple side effects were signi- ficantly higher in the combination group (Table 4). These side effects were transient and the vast majority of patients completed the treatment as per protocol. However, two patients in combination group could not tolerate the side effects after the first hour of nifedipine administration necessitating its discontinuation and they completed atosiban alone. However, their outcomes were included in our ITT analysis. No such intolerability was encountered in the single agent arm. No significant fetal heart rate changes occurred in both arms. The neonatal events were comparable with no significant difference (Table 5).

\subsection{Secondary Outcomes}

The time to delivery was similar between the atosiban (median, 42 days; range, 0.7 - 84 days) and the combination groups (median, 49.7 days; range, 0.7 - 109.2 days). This is reflected in the Kaplan-Meier survival curves showing the relationship between delivery rate and the time to delivery (Figure 2).

There were no statistically significant differences between the two groups regarding the mode of delivery and other secondary outcomes (Table 5).

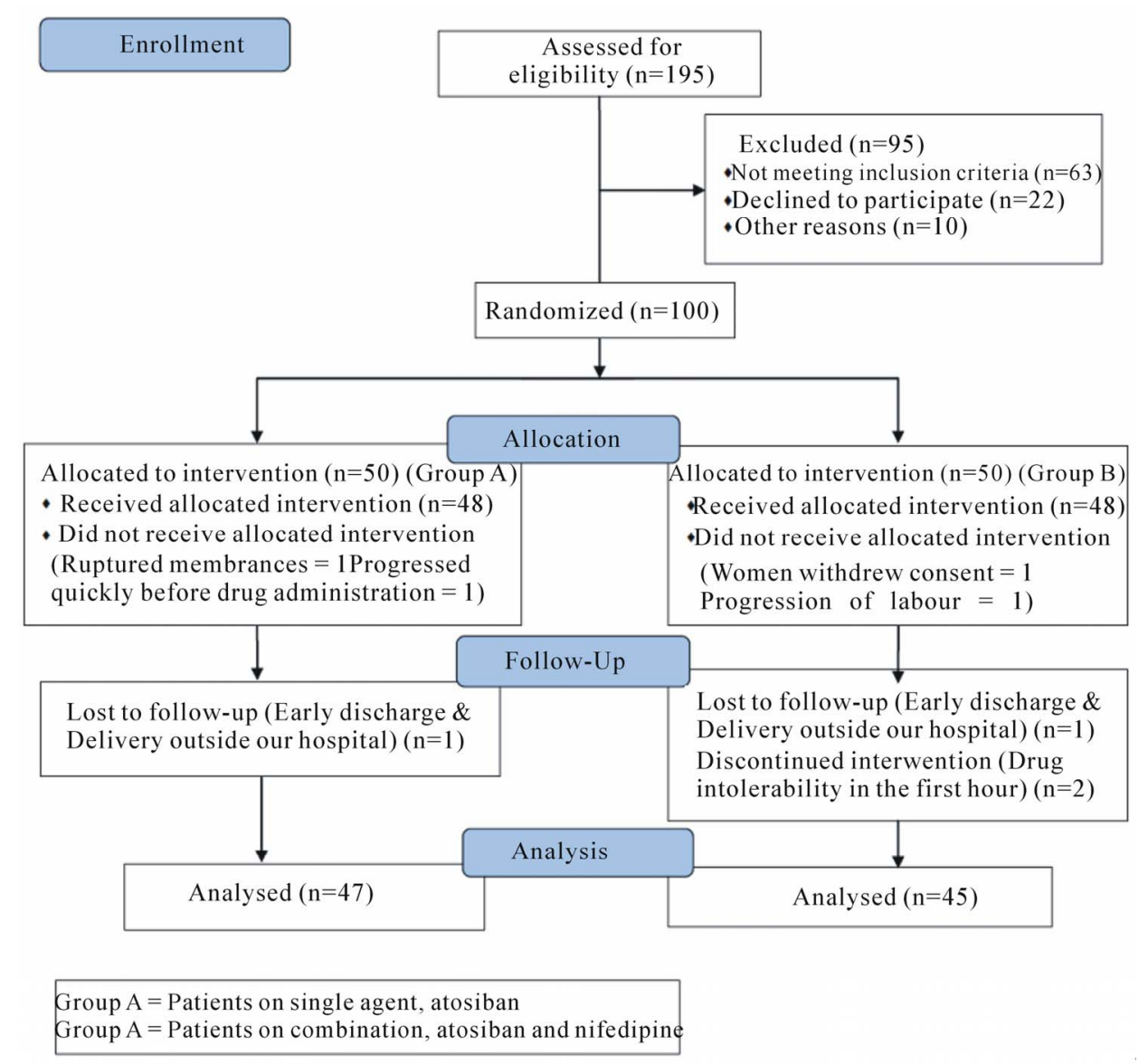

Figure 1. Study profile. 


\section{DISCUSSION}

The incidence of preterm birth has been increasing despite the intense research for prevention [19]. The overall treatment goal of acute tocolysis is to minimize the fetomaternal adverse effects, thus improving long-term family outcomes [20].

Evidence has shown that all current tocolytic agents are superior to no treatment at delaying delivery at both 48 hours and 7 days [21]. However, there was no major impact on perinatal outcomes with acute as well as maintenance tocolysis $[22,23]$. This study explores the clinical utility of combining two tocolytic agents for the treatment of PTL. To our knowledge, this is the first randomized trial conducted in humans comparing the effects of a fixed combination against single tocolytic therapy.

The reasons to choose these two drugs are: their low side effect profile among the available tocolytics $[15,24]$ and our personal experience with atosiban as it has been our standard tocolytic agent since 2003. Their standard dosage was maintained $[14,15]$ as this combination has been shown to have additive effect in animal studies i.e.

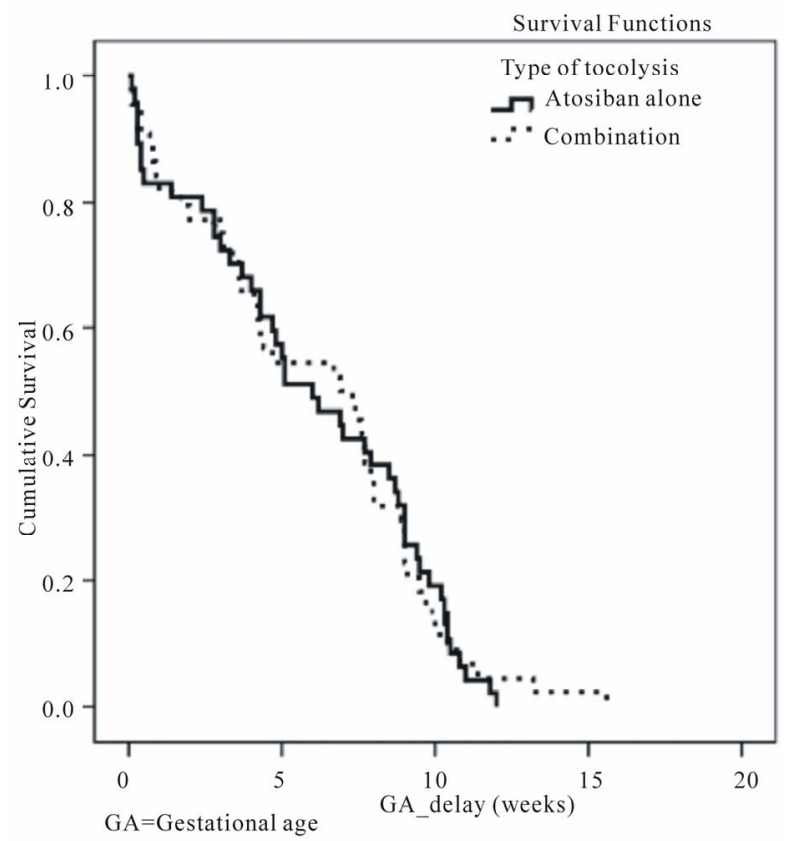

Figure 2. Kaplan-meier curves: gestational age delay since the time of tocolysis.

Table 1. Baseline characteristics of the women according to treatment allocations. Values are given as n (\%), Mean [SD] or median \{Inter Quartile Range\}.

\begin{tabular}{|c|c|c|c|}
\hline & Group A $(n=47)$ & Group B $(n=45)$ & $\mathrm{P}$ \\
\hline Maternal age & $26.3 \pm 5.5$ & $26.5 \pm 5.9$ & 0.87 \\
\hline \multicolumn{4}{|l|}{ Para } \\
\hline 0 & $20(42.6 \%)$ & $27(60 \%)$ & \multirow{2}{*}{0.74} \\
\hline$\geq 1$ & $27(55.3 \%)$ & $18(40 \%)$ & \\
\hline Gestational age at admission (weeks) & $30.42 \pm 2.34$ & $29.90 \pm 2.63$ & 0.42 \\
\hline \multicolumn{4}{|l|}{ Gestational age categories } \\
\hline$<28$ wks & $7(14.9 \%)$ & $7(15.6 \%)$ & \\
\hline $28-32$ wks & $24(51.1 \%)$ & $25(55.6 \%)$ & \\
\hline$>32 \mathrm{wks}$ & $16(34 \%)$ & $13(28.9 \%)$ & \\
\hline Contraction frequency $(\mathrm{n} / 30 \mathrm{~min})$ & $7.9 \pm 2.7$ & $7.9 \pm 2.5$ & 0.70 \\
\hline Cervical dilatation $(\mathrm{cm})$ & $1.5\{1-2\}$ & $2.0\{1-2\}$ & 0.074 \\
\hline
\end{tabular}

Group A = Patients on single agent, atosiban. Group B = Patients on combination, atosiban and nifedipine.

Table 2. Tocolytic efficacy of women of groups A and B at 48 hrs and 7 days and effect of therapy on uterine activity.

\begin{tabular}{cccccc}
\hline & Group A (n=47) & Group B (n=45) & OR & $(95 \%$ CI) & P \\
\hline Tocolytic efficacy & & & & & \\
No failure at 48 hrs & $43(91.5 \%)$ & $41(91.1 \%)$ & 1.05 & $0.25-4.47$ & 0.95 \\
No failure at 7 days & $39(90.7 \%)$ & $36(85.7 \%)$ & 1.63 & $0.42-6.2$ & 0.52 \\
$\begin{array}{c}\text { Hours needed to stop contraction } \\
\text { (mean } \pm \text { SD) }\end{array}$ & $4.0 \pm 2.1$ & $3.1 \pm 1.5$ & - & - & 0.043 \\
\hline
\end{tabular}

Group A = Patients on single agent, atosiban. Group B = Patients on combination, atosiban and nifedipine. 
Table 3. The relationship between tocolytic efficacy of groups A and B with gestational age at 48 hrs and 7 days.

\begin{tabular}{|c|c|c|c|c|c|c|}
\hline \multirow{2}{*}{ Efficacy interval } & \multirow{2}{*}{$\begin{array}{l}\text { Gestational age } \\
\quad \text { (weeks) }\end{array}$} & Group A $(n=47)$ & Group B $(n=45)$ & \multirow{2}{*}{ OR } & \multirow{2}{*}{$(95 \% \mathrm{CI})$} & \multirow{2}{*}{$\mathrm{P}$} \\
\hline & & Proportion (\%) & Proportion (\%) & & & \\
\hline \multirow{4}{*}{$\begin{array}{l}\text { Undelivered } \\
\text { within } 48 \mathrm{hrs}\end{array}$} & $<28$ wks & 6/7 (85.7\%) & $5 / 7(71.4 \%)$ & 2.4 & $0.17-34.93$ & 1.0 \\
\hline & $28-32$ wks & $22 / 24(91.7 \%)$ & 23/25 (92\%) & 0.96 & $0.12-7.40$ & 1.0 \\
\hline & $>32$ wks & $15 / 16(93.8 \%)$ & $13 / 13(100 \%)$ & 0.94 & $0.83-1.06$ & 1.0 \\
\hline & $<28$ wks & $6 / 6(100 \%)$ & $4 / 5(80 \%)$ & 1.25 & $0.81-1.94$ & 0.46 \\
\hline \multirow{2}{*}{$\begin{array}{l}\text { Undelivered } \\
\text { within } 7 \text { days }\end{array}$} & $28-32$ wks & $21 / 22(95.5 \%)$ & $21 / 24(87.5 \%)$ & 3.0 & $0.29-31.23$ & 0.609 \\
\hline & $>32 \mathrm{wks}$ & $12 / 15(80 \%)$ & $11 / 13(84.6 \%)$ & 0.73 & $0.10-5.2$ & 1.0 \\
\hline
\end{tabular}

Group A = Patients on single agent, atosiban. Group B = Patients on combination, atosiban and nifedipine.

Table 4. Frequency and total number of maternal side effects according to tocolytic therapy.

\begin{tabular}{cccc}
\hline & Group A $(\mathrm{n}=47)$ No. (\%) & Group B (n= 45) No. (\%) & P \\
\hline Total no. of women with adverse events & $16(34)$ & $29(64)$ & 0.006 \\
Early drug termination due to adverse events & 0 & 2 & 0.24 \\
Side effects & & & 0.001 \\
Tachycardia & $7(14.9)$ & $22(48.9)$ & 0.001 \\
Hypotension & $7(14.9)$ & $22(48.9)$ & 0.073 \\
Headache & $6(12.8)$ & $2(4.4)$ & 0.96 \\
GI Tract upset & $2(4.3)$ & $1(2.2)$ & 0.49 \\
Chest pain & 0 & & $8(17.8 \%)$ \\
No. of side effects & $11(23.4 \%)$ & $13(28.9 \%)$ & 0.001 \\
One & $4(8.5 \%)$ & $6(13.3 \%)$ & $2(4.4 \%)$ \\
Two & $1(2.1 \%)$ & \\
Three & 0 & & \\
\hline
\end{tabular}

Group A = Patients on single agent, atosiban. Group B = Patients on combination, atosiban and nifedipine.

the observed inhibition of activity was the same as the expected percentage inhibition in an additive pharmacological model [9].

Tocolytic efficacy was measured as the proportion of women who were not delivered and who did not require alternative tocolytic therapy. This end-point is a composite outcome of delay of preterm delivery and drug tolerability. The choice of $48 \mathrm{hrs}$ and 7 days was made mainly because of the time it takes for corticosteroids to have an effect on the fetal lungs [13]. In our study, high proportion of women succeeded to continue with their pregnancy in this time frame following tocolysis (Table 2). However, despite our small sample size, it seems clear that efficacy was comparable between the groups and the addition of nifedipine did not substantially improve the outcome beyond that was achieved with atosiban alone. Efficacy remained comparable even after sub-grouping the patients according to the gestational age (Table 3).

The lack of additional benefits in the combination group might be explained by the fact that, the uterine smooth muscle has already achieved maximal suppression at the recommended dose of atosiban through maximal reduction of intracellular free calcium concentration, so that further reduction is not possible even with the addition of another agent. It has been recognized that although atosiban and nifedipine act at different receptors in the uterus, the ultimate suppression of contractions is achieved by reduction of intracellular concentration of free calcium for both agents [25]. Secondly, the etiologic heterogeneity of PTL adds complexity to the therapeutic approaches [25]. It is conceivable that these undetermined pathological pathways of PTL are difficult to be reversed.

In vitro animal studies have shown that reduction in the integral contractile activity with tocolytic combinations occur faster than single agents $[9,10]$. This effect was also seen in our study, as the uterine quiescence was achieved in significantly shorter duration by the combination group (Table 2 ). This could be related to 
Table 5. Perinatal outcomes according to tocolytic therapy.

\begin{tabular}{lccc}
\hline & Group A (n=47) & Group B (n=45) & P \\
\hline Gestational age at delivery (weeks) & $36.4 \pm 3.3$ & $36.0 \pm 4$ & 0.90 \\
Birth weight (g) & $3480 \pm 1440$ & $3250 \pm 2060$ & 1.00 \\
1-minute Apgar $<$ 7 & $3 / 45(6.7 \%)$ & $6 / 44(13.6 \%)$ & 0.32 \\
5-minute Apgar $<$ 7 & 0 & 0 & 0.64 \\
NICU admission needed & $12(25.5 \%)$ & $14(31.1 \%)$ & 0.24 \\
Duration of stay $>$ 7 days & $11(23.4 \%)$ & $12(26.6 \%)$ & \\
Neonatal death & 0 & 0 & \\
Neonatal morbidity & $10(83.3 \%)$ & $10(66.7 \%)$ & 0.41 \\
Respiratory distress syndrome & $11(91.7 \%)$ & $12(80.0 \%)$ & 0.61 \\
Hyperbilirubinemia & $1(8.3 \%)$ & $2(13.3 \%)$ & \\
Hypoglycemia & 0 & $1(6.7 \%)$ & \\
Bradycardia & $1(8.3 \%)$ & 0.68 \\
Apnea & 0 & $1(6.7 \%)$ & $\mathrm{NS}$ \\
Necrotising enterocolitis & 0 & $2(13.3 \%)$ & $\mathrm{NS}$ \\
Intraventricular hemorrhage & $3(27.3 \%)$ & $6(40.0 \%)$ & 0.49 \\
Septicemia & & & 0.68 \\
\hline
\end{tabular}

Group A = Patients on single agent, atosiban. Group B = Patients on combination, atosiban and nifedipine.

nifedipine as shown in previous studies [8]. This, however, did not translate into further improvement in tocolytic efficacy. Similarly, there were no significant differences in the time to delivery (Figure 2) or in any other secondary outcome measures (Table 5).

The safety of a drug is another factor that will determine the clinical utility. In this study, patients on atosiban alone had significantly fewer side effects compared to the combination group in whom the cardiovascular side effects were especially increased. Also, women in the combination group experienced more than one side effect, some as high as four (Table 4). We believe, these are mainly related to the addition of nifedipine in the combination arm [26], atosiban on its own has a placebo like maternal-fetal side effect profile [24].

In a prospective cohort study, combination tocolysis has been reported to cause severe adverse reactions as high as $2.5 \%$ [27]. However, other studies did not show such serious effects [12]. In these observational studies, multiple courses of different tocolytics were used in clinically critical cases. In our prospective study involving fixed tocolysis in a defined low risk obstetric population, serious side effects were not encountered and the tolerability to combination therapy was generally good. That is despite, two patients in the combination group developed significant hypotension and tachycardia in the first hour of therapy. We used the previously suggested regimen of maximum dose of $40 \mathrm{mg}$ of nifedipine in the first hour [16]. Recent guidelines have recommended an initial oral dose of $20 \mathrm{mg}$ followed by $10-20 \mathrm{mg}$ three to four times daily, adjusted according to uterine activity for up to 48 hours $[28,29]$.
Experimental models have proven the synergistic effects of dual combinations involving mainly $\beta$ (2)-agonists $[9,10]$. However, as the adverse effects of $\beta$ (2)agonists are significant [30], ethical implications need to be considered. In order to decrease the side effects without compromising the tocolytic effect magnitude, delayed rather than simultaneous administration of second tocolytic agent [11] and reduction of the drug dosages have been suggested [9].

Some limitations have to be taken into consideration while analyzing the results of the present study. First, our trial was not blinded. Hence, differential adverse effect profile of two agents could have contributed to potential investigator bias. To minimize this, whenever possible, interpretations were done in an objective manner. For example, maternal symptom of tachycardia was objectively correlated with the heart rate. Second, the restricted availability of participants for the trial contributed to the study focusing on two arms only. The addition of a third arm of single agent nifedipine might have strengthened the study further even though it is recognized now that the tocolytic efficacy of atosiban and nifedipine is comparable and results can be extrapolated as similar $[7,8]$.

\section{CONCLUSION}

This research has addressed the value of combining atosiban and nifedipine in the acute management of PTL. We conclude that the addition of nifedipine did not substantially improve the clinical benefits beyond that was achieved with atosiban alone and also, increases minor 
maternal side effects. Hence, it is not justified to use this combination in clinical practice despite their relatively safe side effect profile. Future research could focus on combination of other tocolytics utilizing differential time of administration and lower doses.

\section{CONTRIBUTION TO AUTHORSHIP}

W. Al-Omari, M. Begam and N. Nagelkerke designed the research. M. Begam and I. Khudhair collected the data. W. Al-Omari, M. Begam and N. Nagelkerke analysed and interpreted data. W. Al-Omari, M. Begam, F. Khan and I. Khudhair drafted the manuscript. N. Nagelkerke performed statistical analysis.

\section{DETAILS OF ETHICS APPROVAL}

The study protocols were approved by Al Ain Medical District Human Research Ethics Committee (Protocol Number of 06/95) dated $4^{\text {th }}$ March 2007.

\section{ACKNOWLEDGEMENTS}

We thank our colleagues at the department of Obstetrics and Gynecology, Tawam Hospital for their cooperation. We are grateful to our patients who accepted to participate in this study.

\section{REFERENCES}

[1] Saigal, S. and Doyle, L.W. (2008) An overview of mortality and sequelae of preterm birth from infancy to adulthood. Lancet, 371, 261-269. doi:10.1016/S0140-6736(08)60136-1

[2] NHMRC (2000) Care around preterm birth. Clinical Practical Guidelines, NHMRC, Australia.

[3] Petrou, S. (2005) The economic consequences of preterm birth during the first 10 years of life. British Journal of Obstetrics and Gynaecology, 112, 10-15. doi:10.1111/j.1471-0528.2005.00577.x

[4] Crowther, C.A., Hiller, J.E. and Doyle, L.W. (2002) Magnesium sulphate for preventing preterm birth in threatened preterm labor. Cochrane Database of Systematic Reviews, Article ID: CD001060. doi:10.1002/14651858.CD001060

[5] Papatsonis, D., Flenady, V., Cole, S. and Liley, H. (2005) Oxytocin receptor antagonists for inhibiting preterm labor. Cochrane Database of Systematic Reviews, 20, Article ID: CD004452.

[6] King, K.J., Flenady, V.J., Papastonis, D.N., Dekker, G.A. and Carbonne, B. (2003) Calcium channel blockers for inhibiting preterm labor. Cochrane Database of Systematic Reviews, Article ID: CD002255.

[7] Kashanian, M., Akbarian, A.R., Soltanzadeh, M. (2005) Atosiban and nifedipin for the treatment of preterm labor. International Journal of Gynecology \& Obstetrics, 91, 10-14. doi:10.1002/14651858.CD001060

[8] Al-Omari, W.R., Al-Shammaa, H.B., Al-Tikriti, E.M. and
Ahmed, K.W. (2006) Atosiban and nifedipine in acute tocolysis: A comparative study. European Journal of $\mathrm{Ob}$ stetrics \& Gynecology and Reproductive Biology, 128, 129-134. doi:10.1016/j.ejogrb.2005.12.010

[9] Doret, M., Mellier, G., Gaucherand, P., Saade, G.R., Benchaib, M. and Frutoso, J. (2003) The in vitro effect of dual combinations of ritodrine, nicardipine and atosiban on contractility of pregnant rat myometrium. British Journal of Obstetrics and Gynaecology, 110, 731-734. doi:10.1111/j.1471-0528.2003.02443.x

[10] Hajagos-Tóth, J., Kormányos, Z., Falkay, G., Pál, A. and Gáspár, R. (2010) Potentiation of the uterus-relaxing effects of $\beta$-adrenergic agonists with nifedipine: Studies on rats and the human myometrium. Acta Obstetricia et Gynecologica Scandinavica, 89, 1284-1289. doi:10.3109/00016349.2010.512064

[11] Nardin, J.M., Carroli, G. and Alfiveric, Z. (2006) Combination of tocolytic agents for inhibiting preterm labor. Cochrane Database of Systematic Reviews, Article ID: CD006169. doi:10.1002/14651858.CD006169

[12] Ingemarsson, I. (2005) Combination therapy. British Journal of Obstetrics and Gynaecology, 112, 89-93.

[13] Moutquin, J.M., Sherman, D. and Cohen, H. (2000) Double blind, randomized, controlled trial of atosiban and ritodrine in the treatment of preterm labor: A multicenter effectiveness and safety study. American Journal of $\mathrm{Ob}$ stetrics \& Gynecology, 182, 1191-1199. doi:10.1067/mob.2000.104950

[14] Goodwin, T.M., Valenzuela, G.J., Silver, H., et al. (1996) Dose ranging study of the oxytocin antagonist atosiban in the treatment of preterm labor. Obstetrics \& Gynecology, 88, 331-336. doi:10.1067/mob.2000.104950

[15] Papastonis, D.N., Van Geijn, H.P., Ader, H.J., Lange, F.M., Bleker, O.P. and Dekker, G.A. (1997). Nifedipine and ritodrine in the management of preterm labor: A randomized multicenter trial. Obstetrics \& Gynecology, 2, 230-234.

[16] RCOG Guidelines No.1 (B) (2002) Tocolytic drugs for women in preterm labor.

http://www.rcog.org.uk/womens-health/clinical-guidance/ tocolytic-drugs-women-preterm-labour-green-top-1b

[17] The Worldwide Atosiban versus Beta-agonists Study Group (2001) Effectiveness and safety of the oxytocin antagonist atosiban versus beta-adrenergic agonists in the treatment of preterm labor. British Journal of Obstetrics and Gynaecology, 108, 133-142.

[18] The Canadian Preterm Labor Investigators Group (1992) Treatment of preterm labor with the beta-adrenergic agent ritodrine. The New England Journal of Medicine, 327, 208-212. doi:10.1056/NEJM199207163270319

[19] Langhoff-Roos, J., Kesmodel, U., Jacobsson, B., Rasmussen, S. and Vogel, I. (2006) Spontaneous preterm delivery in primiparous women at low risk in Denmark: Population based study. BMJ, 332, 937-939. doi: $10.1136 / \mathrm{bmj} .38751 .524132 .2 \mathrm{~F}$

[20] Office for National Statistics (2005) Mortality statistics: childhood, infant and perinatal. Review of the registrar general on deaths in England and Wales. Office for $\mathrm{Na}$ tional Statistics, London. 
[21] Haas, D.M., Imperiale, T.F., Kirkpatrick, P.R., Klein, R.W., Zollinger, T.W. and Golichowski, A.M. (2009) Tocolytic therapy: A meta-analysis and decision analysis. Obstetrics \& Gynecology, 113, 585-594.

[22] Papatsonis, D., Flenady, V. and Liley, H. (2009) Maintenance therapy with oxytocin antagonists for inhibiting preterm birth after threatened preterm labor. Cochrane Database of Systematic Reviews, 21, Article ID: CD005938 .

[23] Lyell, D.J., Pullen, K.M., Mannan, J., Chitkara, U., Druzin, M.L., Caughey, A.B. and El-Sayed, Y.Y. (2008) Maintenance nifedipine tocolysis compared with placebo: A randomized controlled trial. Obstetrics \& Gynecology, 112, 1221-1226. doi:10.1097/AOG.0b013e31818d8386

[24] Romero, R., Sibai, B.M., Sanchez-Ramos, L., Valenzuela, G.J., Veille, J.C., Tabor, B., Perry, K.G., Varner, M., Goodwin, T.M., Lane, R., Smith, J., Shangold, G. and Creasy, G.W. (2000) An oxytocin receptor antagonist (atosiban) in the treatment of preterm labor: A randomized, double-blind, placebo-controlled trial with tocolytic rescue. American Journal of Obstetrics \& Gynecology, 182, 1173-1183. doi:10.1067/mob.2000.95834
[25] Hyagriv, N.S. and Steve, N.C. (2007) Prevention of preterm delivery. The New England Journal of Medicine, 357, 477-487. doi:10.1056/NEJMra050435

[26] Oei, S.G. (2006) Calcium channel blockers for tocolysis: A review of their role and safety following reports of serious adverse events. The European Journal of Obstetrics \& Gynecology and Reproductive Biology, 126, 137-145. doi:10.1016/j.ejogrb.2006.03.001

[27] De Heus, R., Mol, B.W., Erwich, J.J., et al. (2009) Adverse drug reactions to tocolytic treatment for preterm labor: Prospective cohort study. $B M J, 338, \mathrm{~b} 744$. doi:10.1136/bmj.b744

[28] RCOG Guidelines No.1 (B) (2011) Tocolytic drugs for women in preterm labor.

http://www.rcog.org.uk/womens-health/clinical-guidance/ tocolytic-drugs-women-preterm-labour-green-top-1b

[29] British National Formulary. http://bnf.org

[30] Anotayanonth, S., Subhedar, N.V., Neilson, J.P. and Harigopal, S. (2004) Betamimetics for inhibiting preterm labor. Cochrane Database of Systematic Reviews, Article ID: CD004352. 